\title{
Numerical Simulation of Buoyancy-Induced Micropolar Fluid Flow between Two Concentric Isothermal Spheres
}

\author{
M. Khoshab ${ }^{\dagger}$ and A.A. Dehghan \\ Yazd University, Yazd, Yazd, 89195-741, Iran \\ †Corresponding Author Email: masih.khoshab@stu.yazduni.ac.ir
}

(Received April 25, 2010; accepted March 13, 2011)

\begin{abstract}
Natural convection heat transfer between two differentially heated concentric isothermal spheres utilizing micropolar fluid is investigated numerically. The two-dimensional governing equations are discretized using control volume method and solved by employing the alternating direction implicit scheme. Results are presented in the form of streamline and temperature patterns, local and average Nusselt numbers, over the heated and cooled boundaries for a wide range of Rayleigh numbers, Prandtl numbers and dimensionless vortex viscosity $\left(K_{v}\right)$, dimensionless microinertia density $\left(B_{v}\right)$, and microrotation boundary condition $(n)$ for radius ratio of 2 . The goal of this work is to investigate heat transfer characteristics of natural convection in the annulus between concentric spheres using micropolar theory. It is shown that micropolar fluids give lower heat transfer values than those of the Newtonian fluids. It is also found that the average Nusselt number increases with increasing Rayleigh and Prandtl numbers. On the other hand, it is disclosed that increasing the vortex viscosity reduces the heat transfer rate. The results are compared with the data available in the open literatures, and an excellent agreement was obtained. Finally, a correlation between the average Nusselt number, Rayleigh number and material parameter $\left(K_{v}\right)$ is presented.
\end{abstract}

Keywords: Spheres, Natural convection, Micropolar fluid.

\section{NOMENCLATURE}

\begin{tabular}{ll}
$B_{v}$ & Dimensionless material parameter $L^{2} / j$ \\
$C_{p}$ & Specific heat \\
$g$ & gravity \\
$j$ & Microinertia per unit mass \\
$k$ & Thermal conductivity \\
$k_{v}$ & Vortex viscosity \\
$K_{v}$ & Dimensionless Vortex viscosity $k_{v} / \mu$ \\
$L$ & reference length (Gap width $\left.=r_{o}-r_{i}\right)$ \\
$n$ & ratio of the microrotation vector \\
& component and the fluid skin friction at \\
& the wall. \\
$N u$ & Local Nusselt Number. \\
\hline$N u$ & Average Nusselt Number \\
$p$ & pressure \\
$P r$ & Prandtl Number \\
$r$ & dimensionless radial coordinate $\bar{r} / L$ \\
$R^{*}$ & Radius ratio $r_{o} / r_{i}$ \\
$R a$ & Rayleigh Number \\
$\bar{T}$ & Temperature \\
$T$ & Dimensionless Temperature \\
$t$ & time
\end{tabular}

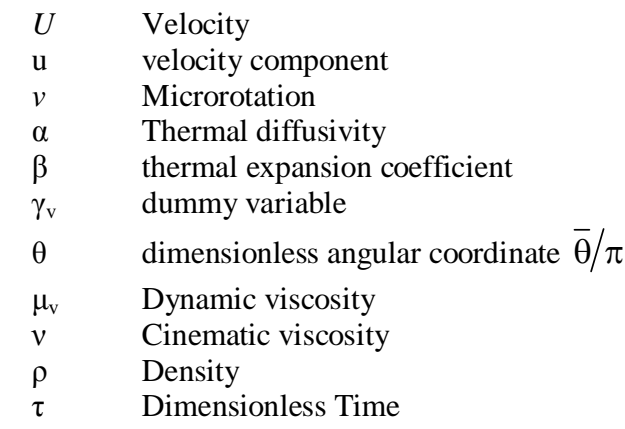

\section{Subscription}

i inner

o outer

$\mathrm{r}$ radial

$\theta \quad$ tangential

ref Reference

w wall condition

$\max \quad$ maximum 


\section{INTRODUCTION}

During recent years, the theory of micropolar fluids has been much investigated and used to describe the characteristics of the fluid flow carrying suspended particles. The theory of micropolar fluid was introduced by Eringen (1964) and has now been applied in the many studies of engineer applications. The concept of such fluids is to provide a mathematical model for the behavior of fluids which are affected by local motions of the material particles contained in each of its volume elements and also taking into account the initial characteristics of the microstructure particles which are permitted to undergo rotation. This local rotation of the particles is independent of the mean fluid flow and its local vorticity field. Later, Eringen (1972) extended this theory to the investigation of thermo-micropolar fluids whose thermal behavior is taken into account. This theory can describe non-Newtonian behavior of certain fluids, such as colloidal fluids, animal blood carrying deformable globules, suspensions and liquids with polymer additives. In recent years, this theory is gaining attention of many researchers who are interested on the developing issues, such as microscale technologies. Papautsky et al. (1999) demonstrated that microchannel fluid flow behavior describing by micropolar theory presents better agreement with experimental data, than with the results obtained by employing classical NavierStokes theory.

Natural convection of a micropolar fluid in a rectangular enclosure was investigated in the work of Hsu and Chen (1996), where they presented a parametric study of the effect of microstructure on the flow and heat transfer. They showed that heat transfer rate and therefore Nusselt number of a micropolar fluid is decreased compared with the Newtonian fluid. Later, Hsu et al. (1997) investigated natural convection of micropolar fluids in an enclosure with a single and or multiple uniform heat sources, where different boundary conditions for microrotation were considered. In their work, heat transfer characteristics and flow phenomenon were presented for different values of the Rayleigh number, enclosure tilting angle and various material properties of the micropolar fluids. The spline alternating direction implicit procedure (SADI) as used to perform the numerical computation. The results indicated that dependence of a microrotation term and heat transfer on a microstructure parameter is significant. They showed that the value of maximum microrotation term $\left(v_{\max }\right)$ increases when either vortex $\operatorname{viscosity}\left(K_{v}\right)$ or microinertia increases $\left(B_{v}\right)$.

Conversely, the value of $v_{\max }$ decreased as the spin gradient viscosity $\left(\gamma_{v}\right)$ was increased. It was observed that the heat transfer rate is quite sensitive to the microrotation boundary conditions $(n)$. Similar works were also published by Aydin and Pop (2005, 2007), where they presented flow patterns for different Rayleigh and Prandtl numbers. They also used some correlations between the coefficients of viscosity and micro-inertia that proposed by Ahmadi (1976). Recently, Zadravec et al. (2009) investigated natural convection of micropolar fluid in a rectangular enclosure with boundary element method and extended the results for Rayleigh number up to the $1 \times 10^{7}$.

On the other hand, natural convection heat transfer in the annulus between two concentric spheres has received much attention due to both theoretical interests and experimental applications. This geometry has been employed in many engineering design problems such as nuclear reactor design, thermal energy storage (TES) systems, solar energy collectors and storage tanks, to name a few. Predicting the transient and steady state behavior of fluid flow and heat transfer rates is a necessary task to these engineering design problems. However, in practice, many of the fluids involved in engineering applications exhibit non-Newtonian behaviors. Consequently, the investigation of natural convection must be extended to the case of nonNewtonian fluids.

To the best of our knowledge, there are only two studies on natural convection heat transfer of micropolar fluid flwo in the annulus between two concentric spheres that conducted by Chiu et al. (1999) and Chen (2005). In the present study, the work of Chiu et al. (1999) are extended to Rayleigh number up to the $1 \times 10^{8}$, including wider range of Prandtl number ranging from 0.1 to 100 , and also for various values of vortex viscosity ratio, $K_{v}$, ranging from 0.0 to 10 . In addition, in the present study effects of microinertia (material parameter, $B_{v}$ ) and microrotation boundary condition $(n)$ are also investigated by using finite volume approach. The results are also compared with the published results of earlier studies (Chen 2005) and very good agreements are observed.

\section{GOVERNING EOUATIONS}

The configuration under study is shown in Fig. 1. Two concentric spheres with radius of $r_{i}$ and $r_{0}$ are considered. The inner sphere having a radius of $r_{i}$ is assumed to be the hot surface with a uniform temperature of $T_{i}$ while the outer one with a radius of $r_{o}$ is the cold surface with a uniform temperature of $\mathrm{T}_{\mathrm{o}}$. Spherical coordinates $(r-\theta)$ are used for the present computation with the origin at the center of spheres. The angular coordinate is measured in the clockwise direction with $\theta=0$ at the top and $\theta=\pi$ at the bottom of spheres.

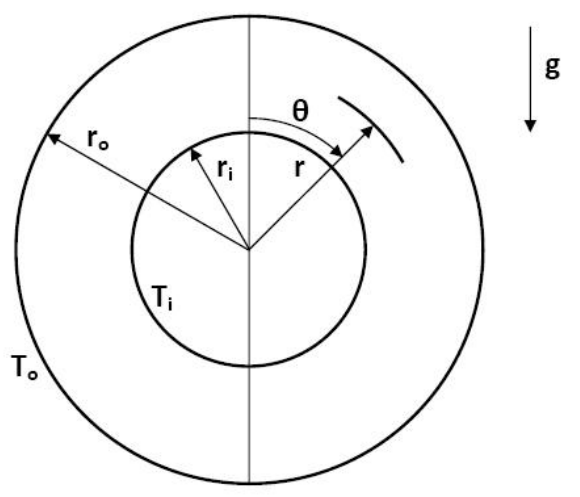

Fig. 1. Physical model and coordinate system. 
The gap between the spheres is assumed to be filled with a micropolar and incompressible fluid. The thermo physical properties of fluid are assumed constant except density variation with temperature in the buoyancy term, i.e. the Boussinesq approximation is utilized. The viscous dissipation term in the energy equation is neglected due to its small influence in natural convection problems with low fluid velocity. For the assumption that micropolar fluid flow will be viscous, incompressible and laminar, the governing equations include conservation laws for mass (1), momentum (2), microrotation (4) and in the case of natural convection also conservation of energy (3) are written as follows.

$\vec{\nabla} \cdot \vec{U}=0$

$\rho \frac{D \vec{U}}{D t}=-\vec{\nabla} p+\left(\mu_{v}+k_{v}\right) \vec{\nabla} \vec{\nabla} \cdot \vec{U}+k_{v} \vec{\nabla} \times \vec{v}$

$+\vec{g} \beta\left(\bar{T}-\bar{T}_{o}\right)$

$\rho C_{P} \frac{D \bar{T}}{D t}=k \nabla^{2} \bar{T}$

$\rho j \frac{D \vec{v}}{D t}=\gamma_{v} \vec{\nabla} \vec{\nabla} \cdot \vec{v}+k_{v} \vec{\nabla} \times \vec{U}-2 k_{v} \vec{v}$

Parameters $\rho, \mu_{v}, k, C_{P}$ and $\beta$ are the density, viscosity, thermal conductivity, specific heat at constant pressure and thermal expansion coefficient of the fluid, respectively, and $k_{v}, \mathrm{j}$ and $\gamma_{v}$ are the vortex viscosity, micro-inertia density, and spin-gradient viscosity of the working fluid, respectively.

Equations (1) - (4) are rewritten in the non-dimensional form for the 2D spherical coordinate as follows.

Continuity equation:

$\frac{1}{r^{2}} \frac{\partial}{\partial r}\left(r^{2} u_{r}\right)+\frac{1}{\pi \cdot r \sin (\pi \theta)} \frac{\partial}{\partial \theta}\left(u_{\theta} \sin (\pi \theta)\right)=0$

Momentum equations:

r-component:

$$
\begin{aligned}
& \frac{\partial u_{r}}{\partial \tau}+u_{r} \frac{\partial u_{r}}{\partial r}+\frac{u_{\theta}}{\pi \cdot r} \frac{\partial u_{r}}{\partial \theta}-\frac{u_{\theta}^{2}}{r}= \\
& \frac{\left(1+K_{v}\right)}{(R a / P r)^{1 / 2}}\left[\nabla^{2} u_{r}-\frac{2}{r^{2}}\left(u_{r}+\frac{\partial u_{\theta}}{\pi \cdot \partial \theta}+u_{\theta} \cot (\pi \theta)\right)\right] \\
& +T \cos (\pi \theta)-\frac{\partial P}{\partial r} \\
& +\frac{K_{v}}{(R a / P r)^{1 / 2}}\left(\frac{1}{r \sin (\pi \theta)} \frac{\partial(\sin (\pi \theta) v)}{\pi \partial \theta}\right)
\end{aligned}
$$

$\theta$-component:

$$
\begin{aligned}
& \frac{\partial u_{\theta}}{\partial \tau}+u_{r} \frac{\partial u_{\theta}}{\partial r}+\frac{u_{\theta}}{\pi \cdot r} \frac{\partial u_{\theta}}{\partial \theta}+\frac{u_{r} u_{\theta}}{r}= \\
& \frac{\left(1+K_{v}\right)}{(R a / P r)^{1 / 2}}\left[\nabla^{2} u_{\theta}+\frac{2}{r^{2}}\left(\frac{\partial u_{r}}{\pi \cdot \partial \theta}-\frac{u_{\theta}}{2 \sin ^{2}(\pi \theta)}\right)\right] \\
& -T \sin (\pi \theta)-\frac{\partial P}{\pi \cdot r \partial \theta}-\frac{K_{v}}{(R a / P r)^{1 / 2}}\left(\frac{\partial(r v)}{r \partial r}\right)
\end{aligned}
$$

Energy equation:

$$
\frac{\partial T}{\partial \tau}+u_{r} \frac{\partial T}{\partial r}+\frac{u_{\theta}}{\pi \cdot r} \frac{\partial T}{\partial \theta}=\frac{1}{(R a / P r)^{1 / 2}}\left(\nabla^{2} T\right)
$$

Microrotation equation:

$$
\begin{aligned}
& \frac{\partial v}{\partial \tau}+u_{r} \frac{\partial v}{\partial r}+\frac{u_{\theta}}{\pi \cdot r} \frac{\partial v}{\partial \theta}=\frac{\left(1+K_{v} / 2\right)}{(\operatorname{Ra} / \operatorname{Pr})^{1 / 2}}\left(\nabla^{2} v\right) \\
& +\frac{K_{v} B_{v}}{(\operatorname{Ra} / \operatorname{Pr})^{1 / 2}} \frac{1}{r}\left[\frac{\partial\left(r u_{\theta}\right)}{\partial r}-\frac{\partial\left(u_{r}\right)}{\pi \partial \theta}\right]-\frac{2 K_{v} B_{v}}{(\operatorname{Ra} / \operatorname{Pr})^{1 / 2}} v
\end{aligned}
$$

Where

$$
\begin{aligned}
& \nabla^{2}()=\frac{\partial^{2}()}{\partial r^{2}}+\frac{\partial^{2}()}{\pi^{2} r^{2} \partial \theta^{2}}+\frac{2}{r} \frac{\partial()}{\partial r} \\
& +\frac{\cos (\pi \theta)}{\pi \cdot r^{2} \sin (\pi \theta)} \frac{\partial()}{\partial \theta}
\end{aligned}
$$

In the foregoing equations, $u_{\theta}$ and $u_{r}$ are the velocity components along the angular, $\theta$, and radial, $r$, directions, respectively. Parameter $\mathrm{T}$ is the fluid dimensionless temperature, and $v$ is the component of micro-rotation whose direction of rotation lies in the $(r-\theta)$ plane. In the above equations, $R a$ is Rayleigh number and $\mathrm{Pr}$ is the Prandtl number of base fluid (pure fluid). Furthermore, the dimensionless parameters $K_{v}$ and $B_{v}$ characterize the vortex viscosity and the microinertia density, respectively, and are defined as:

$$
K_{v}=k_{v} / \mu, B_{v}=L^{2} / j
$$

Where the $L=r_{o}-r_{i}$ is gap width between two spheres. It is assumed that $\gamma_{\nu}$ has the following form as proposed by Ahmadi (1976) and used by Rees and Pop (1998) for the problem of free convection boundary layer flow over a vertical flat plate embedded in a micropolar fluid and by Aydin and Pop (2007) for natural convection in a differentially heated enclosure filled with a micropolar fluid.

$\gamma_{v}=\left(\mu+k_{v} / 2\right) j$

From the non-dimensional form of the governing equations, it is seen that the governing parameters for the present study are the Rayleigh number $(R a)$, the Prandtl number $(P r)$, radius ratio $\left(R^{*}\right)$, the dimensionless parameters $K_{v}, B_{v}$ and microrotation boundary condition, n. If $K_{v}$ is assumed to be zero, Eqs. (6) - (8) reduces to the classical Navier-Stokes equations of Newtonian fluid flow.

The local and average Nusselt numbers are defined as Eqs. (13) and (14), respectively

$$
\begin{aligned}
& N u_{i, o}=-\frac{1}{r_{i} r_{o}}\left[r^{2} \frac{\partial T}{\partial r}\right]_{r=r_{i}, r_{o}} \\
& \overline{N u}=\frac{\pi}{2} \int_{0}^{1} N u_{i, o} \sin (\pi \theta) d \theta
\end{aligned}
$$




\section{The Boundary Conditions}

On the wall surface, the boundary values for $u_{r}$ and $u_{\theta}$ were set to zero, while the temperatures were set to 1 and zero at the hot and cold boundaries respectively. The wall condition for $v$ was specified according to the following relation.

$v_{w}=n \frac{1}{r}\left(\frac{\partial\left(r u_{\theta}\right)}{\partial r}-\frac{\partial\left(u_{r}\right)}{\pi \partial \theta}\right)$

In which $n$ is a constant $0 \leq n \leq 1$. It should be mentioned that the case $n=0$, called strong concentration of suspended particles (Guram and Smith 1980), indicates that concentrated particles close to the rigid boundaries are unable to rotate. The case $n=1$, as proposed by Peddieson (1972) is applied for the modeling of turbulent boundary layer flows. On the other hand, the case $n=1 / 2$, indicates that microrotaion is taken to be equal to the angular velocity at the rigid boundaries. Therefore, the effect of the suspended particles is negligible in the vicinity of the surface because the suspended particles cannot get closer to the surface than their radius. In the vicinity of the surface, rotation of the suspended particles depends only on the fluid shear, so the microrotation vector must be equal to the angular velocity (Ahmadi 1976). In the present study, calculation are performed for $n=1 / 2$, unless it is mentioned elsewhere. The associated boundary conditions on the symmetry axis (i.e. at $\theta=0,1$ ) are as follows.

$\frac{\partial u_{r}}{\partial \theta}=\frac{\partial T}{\partial \theta}=v=u_{\theta}=0$

\section{Solution Procedure}

The properties of the base fluid are evaluated at the reference temperature, i.e. $\mathrm{T}_{\mathrm{o}}$ assumed to be reference temperature $\left(\mathrm{T}_{0}=\mathrm{T}_{\text {ref }}\right)$, the density and the dynamic viscosity are assumed to be constant.

The governing differential equations are solved by employing finite volume method and SIMPLER algorithm described by Patankar (1980). The convective terms, in the momentum, microrotation and energy equations, are discretized using a power-law differencing scheme. Derivatives at the boundaries are approximated by three-point forward or backward differencing formulas. The Alternating Direction Implicit (ADI) method is used for the solution of the discrete equations. The pseudo-transient approach with appropriate under-relaxation parameters for the field parameters is employed for obtaining the steady state results. Non-uniform grids in radial direction and uniform grids in the angular direction are used. Nonuniform grid is generated by using Eq. (17). In order to obtain grid-independent solution for every Rayleigh number, the numerical experimentation is done for four different mesh size configurations. The effect of grid size on the local Nusselt number distribution over the inner and outer walls is investigated (Fig. 2). Therefore mesh sizes of $90 \times 90$ is selected in the present investigation. In most studies, comparison between the maximum values of Nusselt number is chosen in order to verify the grid-independency of the solution while it is believed that comparison between local values is more appropriate and would produce more accurate results. The solution is considered convergent when the global relative errors of the field variables over the all control volume cells are less than prescribed criteria. A convergence criterion of $10^{-7}$ is selected for all field variables except the temperature field for which the value of $10^{-8}$ is considered.The following section outlines general (non-formatting) guidelines to follow. These guidelines are applicable to all authors and include information on the policies and practices relevant to the publication of your manuscript.

$\frac{r_{n}}{L}=\frac{1}{2}\left(1+\frac{\tanh \left(\alpha_{1} \cdot\left(\frac{n}{n_{\max }}-\frac{1}{2}\right)\right)}{\tanh \left(\alpha_{1} / 2\right)}\right)$

Where $3.5 \leq \alpha_{1} \leq 9$.

\section{Results AND Discussion}

Results in the form of flow and thermal field variables and Nusselt numbers are presented for various values of the governing parameters and for a radius ratio of 2 . In order to investigate the accuracy of the numerical procedure developed in this study, typical results were compared with the results available in the open literature.

Recently, the same authors published numerical simulation of buoyancy induced turbulent flow between two concentric isothermal spheres (Dehghan and Khoshab 2010) for Newtonian fluids and various values of Rayleigh numbers ranging from $10^{2}$ to $10^{10}$. Therefore, the comparison of the present results with the results obtained by Chen (2005) is performed only for micropolar fluid flow. Figure 3 presents the average Nusselt number as a function of Rayleigh number for various values of vortex viscosity. The numerical results of Chen (2005) are also presented in the same figure. The results of the present study are extended beyond the previously published data. It is seen that in the convection dominated region, the variation of $\overline{\mathrm{Nu}}$ against $\mathrm{Ra}$ is linear on a double logarithmic scale.

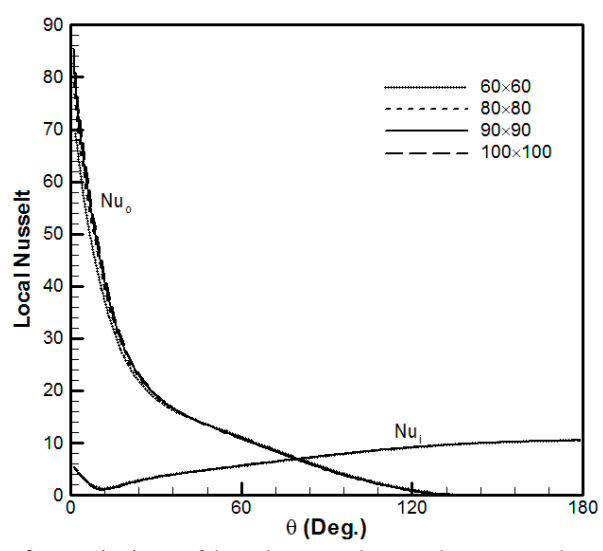

Fig. 2. Variation of local Nusselt number over the cold boundary for various mesh sizes and for $R a=5 \times 10^{7}$, $\operatorname{Pr}=0.71, K_{v}=0.1, B_{v}=1.0$ and $R^{*}=2.0$. 


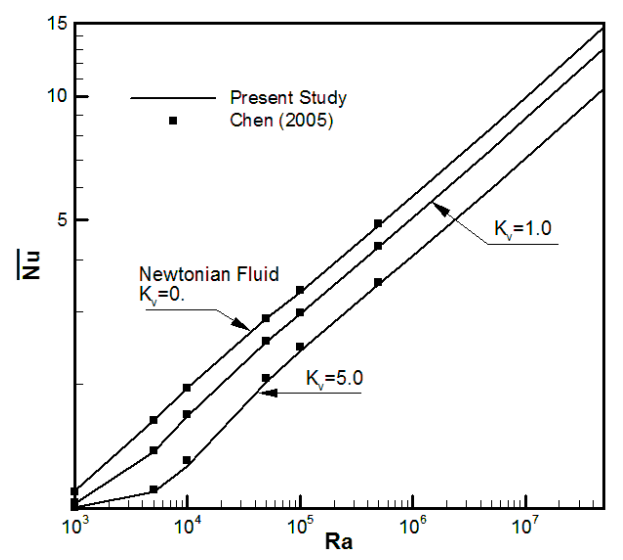

Fig. 3. Variation of average Nusselt number with Rayleigh number for $\operatorname{Pr}=0.7$ and $B_{v}=\gamma_{v}=1.0$.
The heat transfer within the fluid can be characterized by the Rayleigh number value. When the Rayleigh number value is below a certain value, heat transfer is primarily in the form of conduction, when it exceeds beyond that critical value, heat transfer is primarily in the form of convection. The critical value of Rayleigh number is depends on the length characteristic L, Prandtl number Pr and vortex viscosity.

Figure 4 shows the predicted thermal and flow fields in the form of isotherms and streamlines contours for different values of Rayleigh number and dimensionless vortex viscosity. As the flow is symmetric with respect to the vertical axis, the isotherms are plotted on the left and the streamlines are drawn on the right hand of each figure. Increasing the Rayleigh number values intensifies circulation inside the annular cavity.

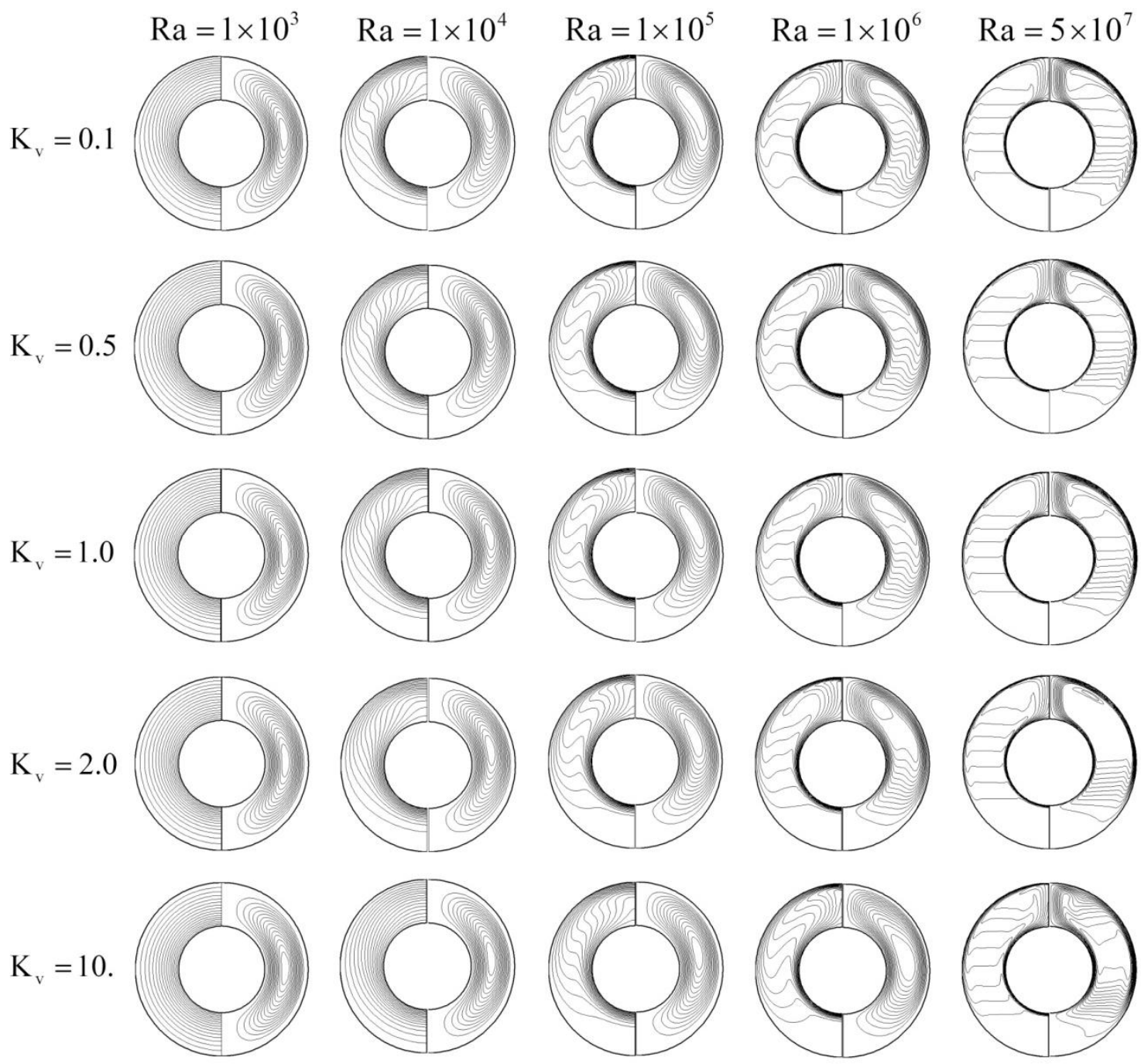

Fig. 4. Isotherms and streamlines for different material parameters $K_{v}$ and different Rayleigh number values

$$
\left(\operatorname{Pr}=0.71, B_{v}=1.0 \text { and } n=0.5\right)
$$

From the Fig. 4 it is also evident that increasing the Rayleigh number value leads to thinner thermal boundary layers near the heated and cooled walls, enhancing momentum and heat transfers in the annular cavity. As the fluid adjacent to the inner wall is heated, the lower density fluid moves upward due to the buoyancy effect, while the relatively colder and denser fluid will eventually flow downward along the cold surface of the outer sphere. Thermal boundary layers start to develop at the lower edge of inner hot wall and the upper edge of upper cold one. The thickness of both boundary layers decreases when the Rayleigh number is 
increased. The thermal boundary layer thickens when the flow moves upward along the inner wall, which results in a decrease of the local heat transfer rate along this wall. The opposite effect is seen adjacent to the outer cold wall when the warm fluid starts to descend along it. For higher values of the Rayleigh number, the boundary layers are confined in the vicinity of the hot and cold surfaces while the core region of the annuli is occupied with a low velocity fluid. It is also observed that for higher values of the Rayleigh number, the bottom portion of the annular cavity is occupied with almost stagnant cold fluid.

As seen for a fixed value of $\mathrm{Ra}$, an increase in $K_{v}$ abates circulation inside the annular cavity and thickens the thermal boundary layers near the heated and cooled walls which lead to momentum and heat transfers reduction, respectively. It is clearly seen that, in the case of $\mathrm{Ra}=1 \times 10^{5}$, as $K_{v}$ increases the thickness of thermal boundary layer increases and the heat transfer rate decreases. In this case, for low values of vortex viscosity ratio, $K_{v}$, intensive inversion can be seen in the isotherms while for higher values of $K_{v}$ the inversion disappears. In the case of $\mathrm{Ra}=1 \times 10^{3}$, for low values of $K_{v}$, both conduction and convection heat transfer mechanisms affect the thermal field while for high values of $K_{v}$, pure conduction heat transfer dominates. As previously mentioned, the critical value of Rayleigh number depends on the vortex viscosity. In the later case, it is seen that, increasing in $K_{v}$ retards the transient regime between the conduction and the convection regime.

This is also true for transient regime between the laminar and turbulent regimes. For each value of Rayleigh number, as $K_{v}$ increases the position of vortex center of the main eddy shift downward and circulation inside the annular cavity alleviates. This is because an increase in the vortex viscosity ratio would result in an increase in the effective viscosity ratio of the fluid flow (see the coefficient of first term in the right hand side of Eq. (6) and (7)).

Figure 5 represents the distribution of local heat transfer coefficients along the hot and cold surfaces for different vortex viscosity ratio, $K_{v}$, and different Rayleigh number values. As can be seen, an increase in $K_{v}$ reduces the heat transfer over the both heated and cooled walls. In the case of $\mathrm{Ra}=10^{3}$, it is also seen that, as $K_{v}$ increases the pure conduction dominates over the walls.

Figure 6 presents the average Nusselt number as a function of Rayleigh number for different material parameters $K_{v}$. It is seen that transition to the convection dominated region depends on $K_{v}$ and increasing in $K_{v}$ retards the transient region. It is also seen that the variation of $\overline{\mathrm{Nu}}$ against $\mathrm{Ra}$ is linear on a double logarithmic scale. As seen for a fixed value of $\mathrm{Ra}$, an increase in $K_{v}$ decreases average Nusselt number. In fact, this is because an increase in the vortex viscosity would result in an increase in the total viscosity of the fluid flow, thus decreasing the heat transfer. The decrease of the heat transfer with the increase of $K_{v}$ is more significant for higher values of Rayleigh number.
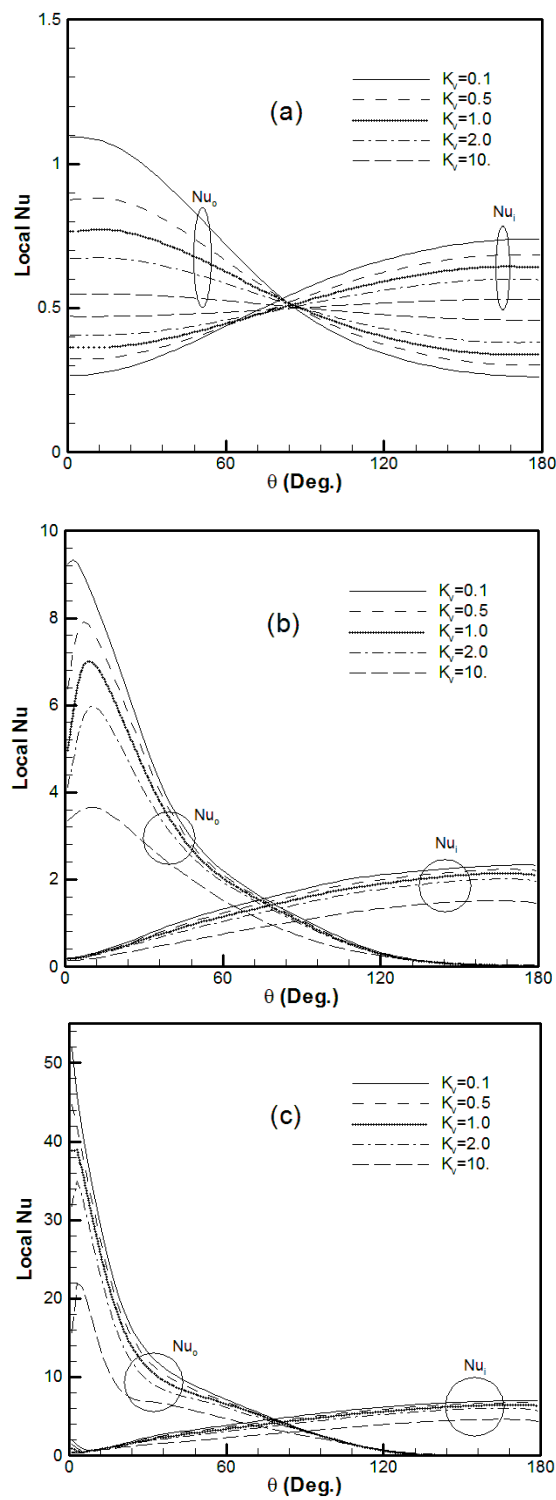

Fig. 5. Distribution of the local Nusselt number for various values of vortex viscosity ratio, $K_{v}$, at inner and outer spheres and for Rayleigh number (a) $10^{3}$, (b) $10^{5}$ and (c) $10^{7}$.

Figure 7 illustrates the effect of the Prandtl number on the heat transfer, for two values of Rayleigh number and $K_{v}=1.0$. It is seen that for fluids with low Prandtl number values $(P r<1), \overline{N u}$ is proportional to both Prandtl number and Rayleigh number i.e. $\overline{N u} \sim(R a \operatorname{Pr})$, while for high Prandtl number value $(P r>1)$ the proportion is in the form of $\overline{N u} \sim(R a)$. Bejan (1984) demonstrated by scale analysis that for fluids with low Prandtl number value $(P r<1)$, the approximate 
expression for the averaged Nusselt number on the vertical flat plate is given in the form of $\overline{N u} \sim(R a \operatorname{Pr})^{1 / 4}$, while in the form of $\overline{N u} \sim R a^{1 / 4}$ for fluids with high Prandtl number value $(P r>1)$. The results obtained here are, therefore, consistent with these above scale analysis.

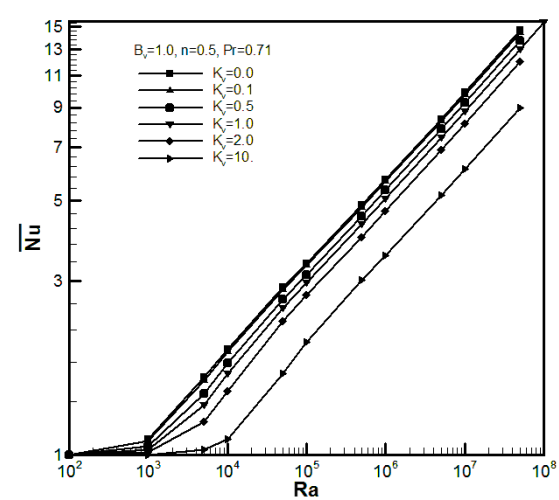

Fig. 6. Variation of average Nusselt number $\overline{N u}$ with Rayleigh number for different material parameters $K_{v}$, $\operatorname{Pr}=0.71, B_{v}=1.0$ and $n=1 / 2$.

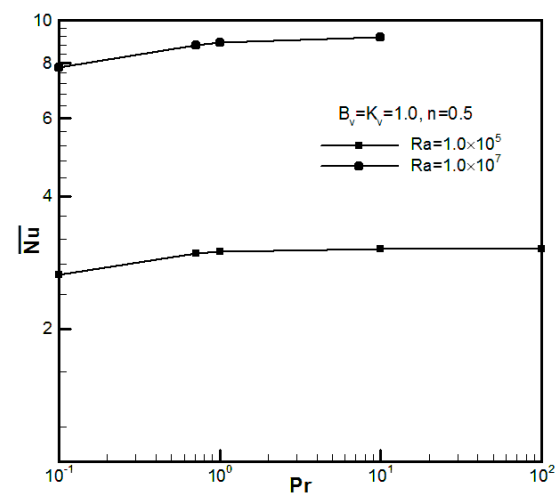

Fig. 7. The effect of Prandtl number, $P r$ on the average Nusselt number, $\overline{N u}$, for $K_{v}=B_{v}=1.0$ and $n=0.5$.

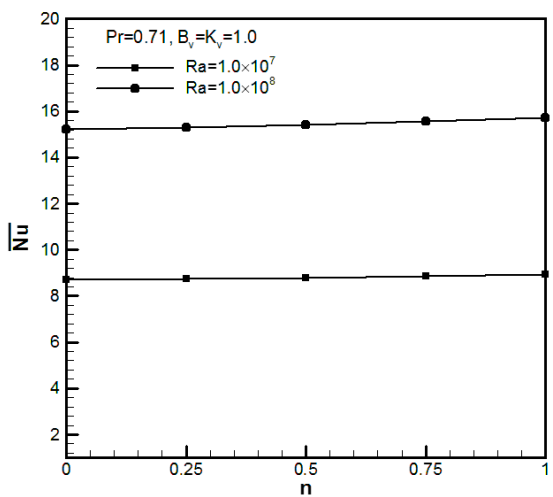

Fig. 8. The effect of microrotation boundary condition on the average Nusselt number, $\overline{N u}$, for $K_{v}=1.0$,

$$
B_{v}=1.0 \text { and } \operatorname{Pr}=0.71 \text {. }
$$

The effect of microrotation boundary condition, $n$, on the heat transfer coefficient is presented in Fig. 8. As microrotation boundary condition increases, a small increase in $\overline{N u}$ is observed. It is because the suspended particles are not free to rotate near the wall for $n=0$, while, the microrotation term is augmented and induces flow enhancement as $n$ increases to 1.0. On the other hand, Figure 9 represents the effect of dimensionless microinertia, $B_{v}$, on the average Nusselt number. It is seen that the parameter $B_{v}$ has not considerable effect on the heat transfer coefficient, $\overline{N u}$ which is in agreement with the result of Hsu et al. (1997) for rectangular cavity.

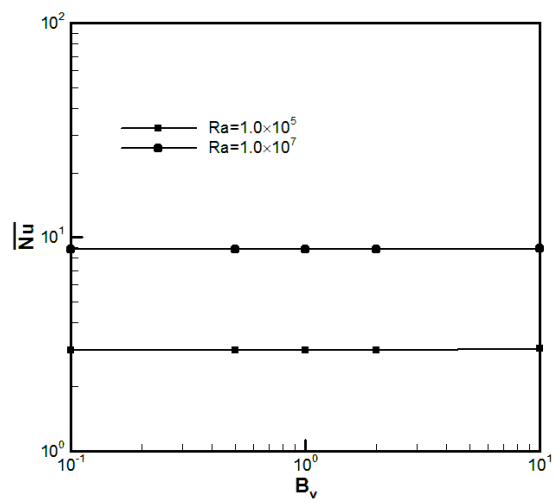

Fig. 9. The effect of dimensionless microinertia $B_{v}$ on the average Nusselt number, $\overline{N u}$, for $K_{v}=1.0$ and

$$
\operatorname{Pr}=0.71 \text {. }
$$

It is seen in Fig. 6 that in the log-log presentation, the $\overline{N u} \sim R a$ relation is almost linear in the laminar flow region. The related equation is

$\overline{N u}=C R a^{m}$

That the average value of heat transfer increases as $R a$ number is increased regardless of vortex viscosity, $K_{v}$, values. The constant values of $C$ and exponent $m$ for Eq. (18) are listed in Table 1 for different values of vortex viscosity, $K_{v}$, in the range of $R a=1 \times 10^{3}-5 \times 10^{7}$.

Table 1 Coefficients of the Eq. (18)

\begin{tabular}{|c|c|c|}
\hline$K_{v}$ & $C$ & $m$ \\
\hline 0.0 & 0.2175 & 0.2372 \\
\hline 0.1 & 0.2133 & 0.2376 \\
\hline 0.5 & 0.2000 & 0.2383 \\
\hline 1.0 & 0.1902 & 0.2380 \\
\hline 2.0 & 0.1790 & 0.2365 \\
\hline 10.0 & 0.1687 & 0.2206 \\
\hline \multicolumn{3}{|l|}{ For $R a=1 \times 10^{3}-5 \times 10^{7}$} \\
\hline
\end{tabular}

Two following simple correlations between the average Nusselt number, $\overline{\mathrm{Nu}}$, Rayleigh number, $\mathrm{R} a$, and vortex viscosity, $K_{v}$, are derived, based on the parametric study conducted in the present investigation.

$\ln \overline{N u}=a+b \ln R a+c K_{v}^{0.5}$
$\ln \overline{N u}=a+b \ln R a+c K_{v}+d K_{v}^{1.5}$ 
Coefficients $a, b, c$ and $d$ in Eqs. (19) and (20) are listed in Table 2. In addition, the goodness of fit value, $R^{2}$, is presented in Table 2.

Table 2 Coefficients of the Eq. (19) and Eq. (20).

\begin{tabular}{|c|c|c|}
\hline & Eq. (19) & Eq. (20) \\
\hline$a$ & -1.527377 & -1.55499 \\
\hline$b$ & 0.238993 & 0.238976 \\
\hline$c$ & -0.156962 & -0.143696 \\
\hline$d$ & $*$ & 0.030109 \\
\hline $\mathrm{R}^{2}$ & 0.99899 & 0.99971 \\
\hline
\end{tabular}

\section{Conclusion}

Natural convection of micropolar fluids in the annulus between two concentric spheres with isothermal cold and hot boundaries has been investigated numerically, using the finite volume method. The results of the present study are extended beyond the previously published data. Simulations are performed to investigate the effects of the Rayleigh number, $R a$, Prandtl number, $P r$ and the material parameters, $K_{v}$ and $B_{v}$, on the momentum and heat transfer. The results are presented in the form of streamlines and isotherms and local variation of Nusselt number over the cold and hot surfaces for various values of the Rayleigh numbers and material parameter $K_{v}$. The results are also compared with the published results of earlier studies and very good agreements were observed. It was seen that for low Rayleigh number, the flow between the concentric spheres has low velocities and exhibits conduction dominated flow and thermal characteristics in both Newtonian and micropolar fluids. Increasing the Rayleigh number changes the flow characteristics to the boundary layer type flow with a relatively high velocity flow adjacent to both surfaces. Furthermore, it is shown that the average Nusselt number increases with increasing Rayleigh and Prandtl numbers. On the other hand, it is disclosed that an increase in the vortex viscosity represented by $K_{v}$, reduces the heat transfer rate. As $K_{v}$ increases the position of vortex center of the eddy shifts downward and circulation inside the annular cavity weakens. This is because an increase in the vortex viscosity ratio would result in an increase in the effective viscosity ratio of the fluid flow. It is also found that, increasing $K_{v}$ retards the transient regime either between the conduction and the convection regimes or the laminar and turbulent regimes.

The numerical results indicate that the average Nusselt number is lower for a micropolar fluid, as compared to a Newtonian fluid. The numerical study performed shows a very significant effect of microstructure on the convective heat transfer rates. Considerable effects on both thermal and velocity fields are found for variation of vortex viscosity. It is observed that the heat transfer rate is less sensitive to the microrotation boundary condition values especially for low values of Rayleigh number. The slight increase in the heat transfer rate for weak concentration flow ( $n=0.5$ or 1 ) is due to the microrotation enhancement. It is concluded that although parameter $B_{v}$ has significant effect on microrotaion field, it has marginal effect on the heat transfer coefficient, $\overline{\mathrm{Nu}}$. Finally, two correlations between the average Nusselt number, Rayleigh number and vortex viscosity are presented.

\section{REFERENCES}

Ahmadi, G. (1976). Self-similar solution of incompressible micropolar boundary layer flow over a semi-infinite flat plate. International Journal of Engineering Science 14, 639-646.

Aydin, O. and I. Pop (2005). Natural convection from a discrete heater in enclosures filled with a micropolar fluid. International Journal of Engineering Science 43, 1409-1418.

Aydin O. and I. Pop (2007). Natural convection in a differentially heated enclosure filled with a micropolar fluid. International Journal of Thermal Science 46, 963-969.

Bejan A., (1984). "Convection Heat Transfer", Wiley, New York.

Chen, W.R. (2005). Transient natural convection of micropolar fluids between concentric and vertically eccentric spheres. International Journal of Heat and Mass Transfer 48, 19361951.

Chiu, C.P., J.Y. Shich and W.R. Chen (1999). Transient natural convection of micropolar fluids in concentric spherical annuli. Acta Mechanica 132, 75-92.

Dehghan, A.A. and M. Khoshab (2010). Numerical simulation of buoyancy-induced turbulent flow between two concentric isothermal spheres. Heat Transfer Engineering 31(1), 33-44.

Eringen, A.C. (1964). Simple microfluids. International Journal of Engineering Science 2, 205-217.

Eringen, A.C. (1972). Theory of thermomicrofluids. J. Math. Anal. Appl. 38, 480-496.

Guram, G.S and C. Smith (1980). Stagnation flows of micropolar fluids with strong and weak interactions. Comput. Math. Appl. 6, 213-33.

Hsu, T.H. and C.K. Chen (1996). Natural convection of micropolar fluids in rectangular enclosure. International Journal of Engineering Science 34(4), 407-415. 
Hsu, T.H., P.T. Hsu and S.Y. Tsai (1997). Natural convection of micropolar fluids in an enclosure with heat sources. International Journal of Heat Mass Transfer 40(17), 42394249.

Papautsky, I., J. Brazzle, T. Ameel and A.B. Frazier (1999). Laminar fluid behaviour in microchannels using micropolar fluid theory. Sensors Actuators 73(1-2), 101-108.

Patankar, S.V. (1980). Numerical Heat Transfer and Fluid Flow. Hemisphere, New York.

Peddieson, J. (1972). An application of the micropolar fluid model to the calculation of turbulent shear flow. International Journal of Engineering Science 10, 23-32.

Rees, D.A.S. and I. Pop (1998). Free convection boundary-layer flow of a micropolar fluid from a vertical flat plate. IMA J. Appl. Math. 61, 179-197.

Zadravec, M., M. Hribersek and L. Skerget (2009). Natural convection of micropolar fluid in an enclosure with boundary element method. Engineering Analysis with Boundary Elements 33, 485-492. 